\title{
Public and Patient Involvement (PPI) in Dementia Research: time to reflect?
}

\author{
Georgina Charlesworth
}

\begin{abstract}
Public and Patient Involvement (PPI) in research is a well-established requirement for applications to many grant-awarding bodies. Numerous models of consultation, collaboration and leadership have been developed to support all stages of research from ideas development through to project execution and dissemination of findings. However, the interface between researchers and lay members is not always a happy one; scientific 'fact' may not square with lived experience; clinical researchers may regard their accumulated knowledge from working with many people with dementia and their families as 'more valid' than the individual experience(s) of a small number of PPI representatives; PPI members can fall victim to tokenism and manipulation. In this opinion piece, I consider the nature of representation in PPI in dementia research, and whether identifying disconfirmatory cases provides greater value than current consensus building approaches. I conclude by encouraging researchers to listen, reflect and embrace the opportunity to engage with alternative perspectives.
\end{abstract}

(151 words)

\section{Background}

My first experience of public and patient involvement (PPI) in research was at an interview for research funding in the late 1990s, when PPI in dementia was in its infancy. The PPI representative on the interview panel had been given a methodological question to read out, complete with hard-topronounce technical terms. Meanwhile the PPI representative who was accompanying me at the invitation of the funder was left to sit outside the interview room without the opportunity to contribute. Fortunately, the research community has come a long way since then in understanding best practice for $\mathrm{PPI}$, and there is now extensive guidance on the 'dos and don'ts' of PPI, such as that produced by NIHR INVOLVE. Since that time I have worked with PPI members on steering and management groups, interview panels, focus groups, stakeholder consultation days, Delphi projects and monitoring visits, for my own projects and those led by others. I strongly believe that PPI can, and does, enhance dementia research. However, not all involvement activities run smoothly. Not all participants approach the PPI enterprise with a spirit of mutual respect. Some researchers tolerate PPI for the sole purpose of ticking a mandatory box on funding applications. Some PPI representatives have developed a 'career' as a paid opinion provider on a wide range of issues on which they have only minimal or vicarious experience. Over the years I have been troubled to witness PPI representatives being silenced, sidelined or ignored where their views do not concur with investigator beliefs, and PPI representatives' views being appropriated, or being misused as ammunition to 'shoot down' alternative perspectives. Most recently, the rise of the young, active, person with dementia as PPI representative can lead to an uncomfortable non-meeting of minds with former carers of parents with advanced dementia and physical frailty.

\section{Who best represents the person with dementia?}


The term PPI is often used synonymously with other nomenclature such as 'service user involvement', 'lived experience experts' and 'experts by experience'. However, the views of 'public' and 'patients' are likely to be very different. Indeed, in the field of dementia, PPI 'representatives' are rarely the peers of research participants, except in the circumstances where the research is with people with early dementia or mild cognitive impairment. Family carers have often been enlisted to represent the voice of the person with dementia. Yet, whilst carers doubtless offer a perspective that is different to, and often invaluable for, researchers, it is not necessarily the current or future perspective of those with the illness. Indeed, where comparisons have been made between self-completion of questionnaires by people with dementia and proxy measures completed by carers, the results consistently demonstrate that the responses of carers and people with dementia are not aligned. Carers' proxy-responses are an approximation of the person with dementia's views and feelings, influenced by the carers' own experience.

If it is not possible for a PPI member to embody representation by being a direct peer of the research participants in question, perhaps they can represent the views and wishes of the wider body of lay people living with an illness or illnesses? Representing the views of a heterogeneous population is not straightforward, due both to the diversity of opinions and the need to set aside ones' own attitudes and beliefs. There are some experienced and dedicated PPI members who have extensive contact with a range of people with dementia and their carers. However, there are certainly researchers who would consider themselves in a similarly strong, if not better, position to represent the 'typical' person with dementia at all stages of impairment for each different dementia diagnosis. For example clinical researchers can draw on years of contact with a sizeable and diverse population of people with dementia and their families within health and social care services, and qualitative researchers can bring to bear skills in identifying and synthesising diverse opinions and perspectives.

Is 'representation' of a PPI perspective the key value of PPI? It could be argued that the 'added value' that PPI brings to research is the lack of indoctrination by the scientific teachings of the specialism under discussion. The thinking of lay members is not constrained by theories or empirical evidence; comments are not limited to 'what research tells us', or 'what theory would predict' but based on a personal perspective derived from life experience. Where researchers engage with the opportunity to explain their thinking to interested, invested, but research-naïve others, and take the time to listen and reflect on the responses, fresh perspectives and lines of enquiry can ensue. Being exposed to alternative perspectives can result in the generation of new ideas, some of which may have immediate benefit whereas others are gems to be developed over the longer term. In working with people with dementia and their carers to develop and evaluate psychological and social interventions, it has been a delight to hear the ideas generated, often as 'throw away' remarks and 'asides' during discussion or tea-break conversations. But how should researchers handle situations where contradiction arises between researcher perspectives (influenced by theory or empirical evidence) and PPI input?

\section{Consensus or confrontation?}

Opposing and oppositional stances often to come to the fore at times when consensus is required and when those involved find that they are being asked to sign up to something to which they cannot agree. Various strategies have been used to work towards consensus, for example nominal group technique and dephi processes. However successfully achieving consensus remains one of the unresolved issues in 
PPI research. Indeed, while describing recent work in developing the revised Guidance for Reporting Patient and Public Involvement (GRIPP2), Brett and colleagues concluded that consensus was only achievable with carefully planning and having a skilled facilitator (Brett et al., 2017). Careful planning includes the clear specification of the intended role of the PPI representatives, ensuring the appropriate skills, support and training for all, and methods for consensus building. Facilitators can help avoid adversarial situations and support negotiations. Aside from guidelines, though, it is not clear that consensus is the only, or indeed the most desirable, outcome for many PPI fora.

Interventions developed on the basis of stakeholder consensus are strongly influenced by customer satisfaction with existing approaches. While having high face validity, the lack of theoretical coherence means that there is often uncertainty over primary outcome and delivery is characterised by low fidelity and poor replication. Rather than seeking consensus between researcher and PPI perspectives, it could be argued that PPI members have a more important role in raising practical and moral concerns, spotting anomalies and flagging up when a proposed course of action would be unacceptable to them. In other words, rather than PPI being focused predominantly on consensus building, PPI may have an important part to play in providing or identifying disconfirmatory evidence. It is disconfirmatory cases that lead to the refinement of existing cases and the generation of new hypotheses.

\section{Conclusions}

Rather than expect PPI members to represent, or be representative of, a position, argument or wider constituency of people with dementia and/or family carers researchers will gain more from embracing the opportunity to listen, question and reflect. Tokenism and manipulation risk giving researchers a bad name, but this is a reason to question the practices of individual researchers and not the PPI enterprise. PPI in dementia may be enhanced by funders and researchers being more explicit in their conceiving of $\mathrm{PPI}$, the contribution that different groups make, and the methods employed.

\section{References}

Brett, J., Staniszewska, S., Simera, I., Seers, K., Mockford, C., Goodlad, S., ... \& Entwistle, A. R. (2017). Reaching consensus on reporting patient and public involvement (PPI) in research: methods and lessons learned from the development of reporting guidelines. BMJ open, 7(10), e016948.

\section{Biography}

Georgina Charlesworth is a senior lecturer in University College London, and a consultant clinical psychologist in North East London NHS Foundation Trust. She has over 25years experience working with people with dementia and families as stakeholders in developing and evaluating interventions such as $\mathrm{CBT}$, befriending and peer support. 